\title{
Paradoxes and Pitfalls of Interleukin-33 in Atherosclerosis
}

\author{
Pavel Kunes ${ }^{1}$, Zdenka Holubcova ${ }^{1}$, Martina Kolackova ${ }^{2}$ and Jan Krejsek ${ }^{2, *}$ \\ ${ }^{I}$ Department of Cardiac Surgery, Charles University in Prague, Medical School and University Hospital in Hradec \\ Kralove, Czech Republic \\ ${ }^{2}$ Department of Clinical Immunology and Allergology, Charles University in Prague Medical School and University \\ Hospital in Hradec Kralove, Czech Republic
}

\begin{abstract}
Atherosclerosis is definitely considered as an inflammatory/immunopathological disease. The long-standing low-grade inflammation is focused on the components of the vessel wall. Initially, this inflammation was assumed to be driven by the pro-inflammatory Th1 cellular and cytokine immune responses. On the basis of accumulating knowledge, however, this view has been specified to include the Th17/Th1 axis which underlies most immunopathological diseases accompanied by sterile inflammation. On the other hand, an anti-inflammatory Th2 cellular and cytokine immune response attempts to dampen these unfavorable reactions which terminate in full-blown atherosclerosis. Interleukin-33, the novel member of the IL-1 cytokine superfamily, was suggested to take part in the anti-atherogenic response by mediating the Th1-to-Th2 switch of the immune reactions. However, IL-33 is a multifaceted mediator with both pro- and antiinflammatory activities. IL-33 presents both an extracellular (cytokine-like) and a nuclear-bound (transcription factor-like) form, each of them performing distinct activities of their own. This review article summarizes latest data relevant to IL33 's role in atherosclerosis, underscoring the paradoxes and pitfalls of laboratory findings and their extrapolation to live organisms including humans.
\end{abstract}

Keywords: Interleukin -33, ST2 receptor, atherosclerosis, inflammation, lipid metabolism, gene transcription, Th1-to-Th2 transition.

\section{ATHEROSCLEROSIS AS AN INFLAMMATORY DIS- EASE}

Atherosclerosis is initiated by the retention in the vascular subendothelial space of atherogenic lipoproteins. From the biophysical viewpoint, disease-susceptible segments of the arterial tree are discerned by the exposure of their inner wall to disturbed blood flow. Vascular sites menaced by atherosclerosis exhibit incongruences of the vessel lumen, such as the aortic root or the carotid bulbs, or by meandering course of the vessels, such as the coronary arteries whose actual shape alternates with each heart beat. Autopsy findings in children who died of accidents revealed that lipoprotein retention is the first step in atherogenesis. It precedes and evokes local vascular inflammatory response. Having entered the vascular wall, atherogenic lipoprotein particles, such as low-density lipoprotein (LDL) and very-low density lipoprotein (VLDL), are retained within the extracellular matrix. This entrapment occurs by the interaction of negatively charged sulphate groups of matrix proteoglycans and positively charged domains of apolipoprotein B-100. LDL particles within the arterial wall are highly susceptible to oxidation and acetylation. Oxidative and enzymatic attacks of the LDL particles underlie the formation of proinflammatory phospholipids and lipid peroxides. Apo B-100

*Address correspondence to this author at the Department of Clinical Immunology and Allergology, Charles University in Prague, Medical School and University Hospital in Hradec Kralove, Czech Republic;

Tel: 420495832270; Fax: 420495833454; E-mail: krejsek@fnhk.cz undergoes fragmentation, which generates aldehyde and phospholipid adducts to apo B-derived peptides. These products evoke an inflammatory response, which proceeds by endothelial expression of adhesion molecules leading to persistent recruitment of neutrophils, monocytes and $C D 4^{+}$ Th1 lymphocytes to nascent atherosclerotic lesions. These cells provide a basis for the inflammatory infiltrate, which is quickly supplemented by mast cells and dendritic cells. Monocytes transform into resident macrophages, which make an effort to remove from the vascular wall all cytotoxic particles, most importantly LDL-derived cholesterol [1].

In early stages of atherogenesis, the prominent macrophage activity is seen in foam cell formation. This process is characterized by ingestion and metabolism of LDL-derived cholesterol. Intracellularly, cholesterol is trafficked from late endosomes to the endoplasmic reticulum, each cell's factory, where the enzyme acyl-CoA/cholesterol acyl transferase (ACAT) esterifies ingested cholesterol to cholesteryl fatty acyl esters. Cholesteryl esters are less cytotoxic than free cholesterol. Membrane-bound intracellular lipid droplets give a foamy appearance to the cells. Foam cells are not passive cholesterol reservoir, but they do concurrently secrete pro-atherogenic molecules, e.g. pro-inflammatory cytokines, by which they contribute to the furtive extension of nascent atherosclerotic plaques [2]. This process is perpetuated by multiple cytokines, the most important being interleukin-12 (IL-12) and interferon- $\gamma(\mathbf{I F N}-\boldsymbol{\gamma})$. Both are typical Th1 immune response cytokines. Atherogenesis driven by the Th1 immune response has been extensively reviewed 
elsewhere and the reader is kindly asked to refer to these fundamental papers [3-5]. Simultaneously, a protective antiinflammatory Th2 immune response sets in. Although less well explored than the former, it incontestably modifies the course of atherosclerosis, most effectively its early stages. To render the issue even more complicated, it should be emphasized here that any immune reaction, irrespective of its formal classification, may turn out, in keeping with actual environmental cues, either pro- or anti-inflammatory or, most surprisingly, it may act both ways at the same time [6]. To shed some light on this perplexity, we now attempt to deal with the case of interleukin-33 in atherogenesis.

\section{ESSENTIAL BIOLOGY OF IL-33}

Interleukin-33 (IL-33) is the latest accrual to the IL-1 cytokine superfamily. Altogether, this superfamily encom-

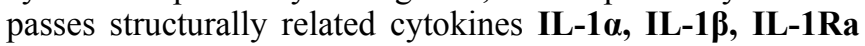
(IL-1 receptor antagonist), IL-18 and IL-33. IL-1 $\beta$ and IL-18 are rated as primordial inflammatory cytokines, whereas IL$1 \mathrm{Ra}$ is an anti-inflammatory peptide which dampens excessive activities of IL-1 $\beta$. IL-33, in its own turn, stands out as a cytokine with dual function. Through the activation of the ST2L membrane receptor complex it acts as a traditionally conceived cytokine. On the other hand, IL-33 performs the role of a nuclear transcription factor. Originally, IL-33 was recognized as a nuclear factor of high endothelial venules [7]. In 2005, Schmitz and coworkers reported that this $n u$ clear factor exhibited a striking amino acid homology with the cytokine IL-18 [8].

Both IL-1 $\beta$ and IL-18 are synthesized as biologically inactive precursors. Under inflammatory conditions, they are activated by cleaving via caspase-1. Initially, it was assumed that IL-33 underwent an analogous caspase-1-mediated processing. However, recent findings revealed that such a proteolytic handling was not required for IL-33 cytokine signaling [9]. By contrast, modification of the full-length IL-33 molecule by caspases mediates its inactivation in steady state conditions when apoptosis is the prevailing mechanism of tissue turnover and no need exists for alerting inflammation [10].

Whenever homeostasis is disrupted, such as is the case of mechanical trauma including surgical operations, infection or cellular stress of any origin, cellular necrosis of various extent enters the stage. IL-33 is now released from necrotic cells which underscores its function of an alarmin alerting the immune system [11]. Collectively, it may be summarized that:

1. When body homeostasis is maintained, apoptosis takes care of tissue integrity by eliminating senescent and dysfunctional cells. IL-33 originating mostly from this tissue debris is cleaved by caspases to silence its proinflammatory activities.

2. When homeostasis has lost equilibrium by any harmful stimulus, full length - biologically active - IL-33 is released from necrotic cells to serve as an endogenous danger signal or "alarmin".

The "split personality" character of IL-33 is recapitulated in the ST2 receptor gene which, by alternative splicing, encodes for two isoforms of the protein [12]:
1. a membrane-bound ST2L isoform which accomplishes the IL-33-borne signal transmission through a receptor complex; this is a heterodimer made up by ST2L + IL$1 \mathrm{RAcP}$ (IL-1R accessory protein), the latter being a subunit shared by other members of the IL-1 cytokine family,

2. a secreted soluble ST2 (sST2) isoform which is devoid of IL-1RAcP and therefore impedes signal transmission; it serves as a decoy receptor for IL-33 switching off its biological effects.

On the target cell membrane level, there is at least one more regulatory molecule, which is known as SIGIRR (single Ig IL-1R-related molecule). It also down-regulates signal transmission by IL-33 [13].

Under steady-state conditions, when no danger to the host has been sensed, IL-33 is expressed in various human tissues, particularly those which are in contact with environmental conditions, either external, such as the skin, or internal, such as the gastrointestinal tract and the lungs. Most importantly from our viewpoint, IL-33 expression has been documented in coronary artery endothelium, coronary artery smooth muscle cells, cardiac fibroblasts, and adipocytes [14].

\section{EARLY ATHEROSCLEROSIS: CAN IT BE STOPPED OR REVERSED BY IL-33?}

Inflammatory cell infiltration of still healthy, but atherosclerosis-susceptible vascular territories begins with CD4+ T lymphocytes. Only later are they followed by monocytes from the blood flow while from the opposite direction, i.e. the media, smooth muscle cells slowly migrate to join the former in the intima. This initial inflammatory cellular network is complemented by mast cells and by dendritic cells, the latter entering intima from the adventitia. Thus, a prerequisite is established for a local immune reaction in the arterial wall, beginning in the intima [15]. The inflammatory nature of these earliest vascular changes is beyond any doubt since resident macrophages do not yet exhibit appearance of foam cells, just the same as lipid deposits are not yet detectable in the intima. Only in subsequent evolution, but still in young and incontestably healthy persons, plasma lipoproteins are retained in the subendothelial layer to be engulfed by macrophages and, to a lesser degree, by new-coming smooth muscle cells. Both of these cell populations can transform into foam cells. As the process goes on, the increasingly accumulating foam cells convert into fatty streaks. A morphological basis for future atherosclerotic plaques is being formed [16]. At least up to this stage, the disease might be slowed down or even reversed by antiinflammatory reactions. One of their branches is represented by the IL-33/ST2 axis.

To get an insight into early human atherogenesis, which previously yielded its secrets only from autopsies of very young people dying mostly by accidents, the disease development could be extensively studied with the advent of genetically modified mice. From among these animal models, $\mathrm{ApoE}^{-/-}$mice lacking apolipoprotein $\mathrm{E}$ all by themselves mimic the course of events taking place in human atherosclerosis. When exposed to selected disease-promoting or, alter- 
natively, disease-protective environment, these animals recapitulate the impact on atherosclerosis of respective conditions encountered in life. Quite recently, ST2 $2^{--}$mice have been generated which by analogy miss the IL-33 membrane receptor ST2L. These live models can yield additional indepth information concerning the influence of the IL-33/ST2 axis, which were so far accessible only from in vitro cellular experiments. Thus, it has been definitely evidenced that the IL-33/ST2 axis, previously suggested to play exclusive antiinflammatory and anti-atherogenetic roles, is endowed with the potential to act both ways, either in a pro- or in an antiinflammatory fashion, in accordance with actual environmental and cellular context [17]. This finding provides yet another piece of knowledge maintaining that atherosclerosis is not a priori doomed to unrestrained progression, but a regression of varying extent and duration might be possible.

\section{IL-33 AND ATHEROPROTECTIVE NATURAL IGM ANTIBODIES}

In vitro, IL-33 has been shown to constrain macrophage foam cell formation by decreasing modified (i.e. acetylated or oxidized; AcLDL or OxLDL) low-density lipoprotein uptake. Both total and esterified macrophage cholesterol content is thereupon reduced, whereas cholesterol efflux out of the cell is increased and ready to undergo the so-called reverse cholesterol transport from the vessel wall into the liver. These IL-33-mediated effects result for the most part from genetic reprogramming of the target cell, a process which translates into decreased expression of genes involved in LDL uptake, such as CD36, and into an increase in genes responsible of cholesterol efflux, such as Apo E. The atheroprotective capacity of IL-33 has been recapitulated in vivo in Apo $\mathrm{E}^{-/}$mice on lipid-rich diet with concomitant administration of IL-33 by an intraperitoneal route. In these diseaseprone animals, IL-33 has been found to decrease the total number of vessel wall-associated $\mathrm{F} 4 / 80^{+}$macrophages, i.e. those cell lines which otherwise could be converted into foam cells [18]. This IL-33-mediated effect is reminiscent of the clearance by apoptosis of lipid-accumulating macrophages, a process that naturally occurs in early stages of atherosclerosis [19]. At the same time, $\mathrm{ApoE}^{-/-}$mice that are treated with IL-33 produce higher titers of anti-ox-LDL IgM antibodies which are assumed to display atheroprotective activities. Natural IgM antibodies, i.e. pre-existing antibodies that arise without previous antigen encounter, are produced by $\mathbf{B 1} \mathbf{B}$ lymphocytes [20]. This fact enables them to exert a non-redundant function in the first-line defense against invading pathogens. Furthermore, natural $\operatorname{IgM}$ antibodies are reactive against neo-self-structures or modified self-antigens that are characteristic of body waste, most prominently necrotic and apoptotic cells. These housekeeping functions promote the clearance of damaged cells and cellular debris whose excessive accumulation would precipitate immune responses against self-constituents as structurally altered self-components are recognized as "danger signals" by innate immune receptors [21]. Given these properties, it is conceivable that natural IgM antibodies play a protective role in atherosclerosis. More specifically, the natural IgM antibody of the T15/EO6 clonotype offers protection from the development of chronic inflammatory lesions through the recognition of stress-induced self-structures such as OxLDL.
Indeed, T15/EO6 IgM has the capacity to block the uptake of OxLDL by the macrophage scavenger receptors CD36 and SRB-1, thereby preventing the formation of inflammatory foam cells, which is a rate-limiting step in atherogenesis [22]. Apoptotic cells display prominent oxidation-specific structures. Consistent with the high abundance of oxidation epitope-specific IgM in the sera of B-1 cell reconstituted mice, it has been shown that the entire set of B-1 cell-derived IgM bound to the surface of apoptotic cells [23]. Aside from the clearance of apoptotic cells and cellular debris, natural IgM antibodies also possess an important protective role by preventing inflammatory reactions induced by the same oxidized lipids that they recognize. Apoptotic cells or blebs carrying oxidation-specific epitopes have the capacity to activate endothelial cells resulting in increased adhesion of monocytes to them [24]. The T15/EO6 IgM antibodies are capable of completely blocking this inflammatory reaction on endothelial cells, an event that likely occurs in various settings in vivo [25]. It has been reported that IL-33 activates B1 cells, which express the IL-33 receptor ST2 [26]. IL-33 markedly activated B1 cell proliferation and enhanced the production of anti-inflammatory and anti-atherogenic mediators IgM, IL-5, and IL-13 in vitro and in vivo in a ST2dependent manner [18]. Whether this potentially preventive phenomenon may be applied to human atherogenesis remains to be established.

Moreover, a substantial increase is encountered in plasma concentrations of anti-inflammatory cytokines IL-4, IL-5, and IL-13, all of them evidencing a Th1 $\rightarrow$ Th2 immune response skewing. Of note, IL-5 is implicated in the generation of atheroprotective antibodies [27]. Concomitantly, expression of the paramount Th1 pro-atherogenic cytokine IFN- $\gamma$ is decreased. In its turn, IFN- $\gamma$ mightily supports the engulfment of LDL by macrophages and the conversion thereof into foam cells while it restrains macrophage cholesterol efflux [28]. This impact of IL-33 is detectable not only in the vascular compartment, but also in adjacent lymph nodes.

\section{IL-33 AND THE RECEPTOR ST2 IN ATHEROSCLE- ROSIS}

The involvement of the two isoforms of the ST2 receptor and their mutually antagonistic impact on the IL-33 response should be reminded here. The expression of ST2L, the membrane-bound form of IL-33 receptor on macrophages, is a sine qua non for this cytokine's atheroprotective effects. Actually, in ST2 ${ }^{-1-}$ macrophages that are completely devoid of this receptor protein, IL-33 by itself can neither decrease the uptake of LDL nor reduce intracellular cholesterol levels. This fact holds true even for ST2 ${ }^{-/-}$foamy cells which have not yet replenished all of their lipid-storing capacity. Moreover, the magnitude of LDL uptake in quiescent, unstimulated ST2 ${ }^{-/-}$macrophages is substantially higher than that in their wild-type, i.e. ST2 ${ }^{+/+}$counterparts. It can thus be inferred that this particular receptor isoform contributes to a certain degree of autoregulation of LDL uptake by macrophages. Conceivably, IL-33 supports cholesterol efflux from wild-type, ST2 ${ }^{+/+}$macrophages, but it is unable to display any effect on ST2 $2^{-/}$cells. Furthermore, IL-33 was found to decrease mRNA and protein expression of scavenger receptors implicated in LDL cellular uptake, such as CD36, SR-A, and SR-B1. At the same time, IL-33 is able to up-regulate 
mRNA and protein expression of cholesterol-depleting mediators ApoE and ABCA-1. These effects are apparent in murine wild-type $\mathrm{ST}^{+/+}$macrophages, but are virtually absent in $\mathrm{ST}^{-/-}$cells. By contrast, Apo $\mathrm{E}^{-/-}$mice treated with intraperitoneal injections of $\mathbf{S S T 2}$, the decoy receptor which is proposed to switch-off the effects of IL-33, were shown to develop significantly larger atherosclerotic plaques and increased plasma IFN- $\gamma$ levels in comparison with control animals. The aforementioned effects of IL-33 on cholesterol traffic into and out of the cell, respectively, and on the resulting development of foam cells, are precisely the opposite to those seen either with $\mathrm{SST} 2$ or IFN- $\gamma$ [18].

\section{IL-33 AND GENE EXPRESSION IN ATHEROSCLE- ROSIS}

As has been hinted above, the particular mechanism accounting for IL-33 activities in the vessel wall can be traced down to modified expression of key genes that are implicated in cholesterol trafficking. The internalization by macrophages of modified LDL particles is dependent on scavenger receptors, such as SR-A, SR-B1, and CD36. Conversely, cholesterol efflux from macrophages is carried out by a mechanism known as reverse cholesterol transport. This is an active process which is executed by members of the ABC transporter family, such as ABCA-1 and ABCG-1, and which requires the presence of lipid-free cholesterol acceptors, such as ApoE and ApoA-I. IL-33 is endowed with the capacity to down-regulate both mRNA and protein expression in human monocyte-derived macrophages of SR-A, SR$\mathrm{B} 1$ and $\mathrm{CD} 36$ and, in parallel, to increase mRNA and protein expression of ApoE, ABCA-1, and ABCG-1 in the same cell lines. Moreover, IL-33-induced increase in ApoE, ABCA-1, and ABCG-1 expression has been documented even in LDLconverted ST2 ${ }^{+/+}$foam cells [29].

Intracellular lipid accumulation underlying macrophage foam cell transformation involves not only an imbalance in the uptake and the efflux of modified LDL particles and of cholesterol, respectively, but distinct processes supporting intracellular storage of cholesteryl esters and triglyceriderich lipid droplets are active with no less importance. Once again, IL-33 is highly operative on this cytoplasmic level. As can be inferred from what has been said so far, IL-33 significantly reduces total and esterified cholesterol content in human monocyte-derived macrophages and even in macrophage-derived foam cells. Intracellular mechanisms controlling cytoplasmic volume of cholesteryl esters and triglyceride-rich droplets are regulated by a network of proteins, the activity of which is in their turn cytokine-sensitive. Among others, following constituents are of importance:
i. Niemann Pick type C (NPC-1 and NPC-2),
ii. carnitine palmitoyltransferase-1 (CPT-1),
iii. adipocyte differentiation-related protein (ADRP),
iv. acyl-CoA:cholesterol acyltransferase-1 (ACAT-1),
v. neutral cholesteryl ester hydrolase (NCEH).

Their respective functions can be briefly summarized as follows: a. NPC-1 and NPC-2 control cholesterol trafficking from the late endosome/lysosome compartment to the plasma membrane, thus regulating cholesterol efflux [30],

b. CPT-1 decreases the availability of fatty acids for cholesterol esterification [31],

c. ADRP increases triglyceride storage [32],

d. ACAT-1 in co-operation with macrophage-derived foam cells, esterifies cholesterol within the endoplasmic reticulum [33],

e. NCEH impacts cholesteryl ester accumulation by regulating its hydrolysis [34].

IL-33 significantly increased NPC-1 and NPC-2 mRNA expression. These results are consistent with the higher levels of cholesterol efflux and mRNA/protein expression of ApoE, ABCA-1, and ABCG-1 in human monocyte-derived macrophages.

IL-33 also increased CPT-1 mRNA expression suggesting that it contributes to less cholesterol esterification. Furthermore, IL-33 significantly decreased ADRP and ACAT-1 mRNA expression. The reduction in these two genes may contribute to less cholesteryl ester accumulation and triglyceride storage. IL-33 also significantly reduced NCEH mRNA expression although this observed effect is not consistent with IL-33 stimulated changes in cholesteryl ester levels because over-expression of NCEH reduces cholesteryl esters in human macrophages. Collectively, these data indicate that IL-33

i. reduces the levels of total and esterified cholesterol content in human macrophages

ii. this particular phenotype involves the alteration in the expression of genes that are implicated in cholesterol storage and transport.

These effects of IL-33 are inseparably associated with the presence of its membrane receptor ST2L on the target cells, whereas they are annihilated by the soluble form of the receptor sST2 [18].

Overall, it can be soundly assumed that the effect of IL33 on ox-LDL-inflicted tissue damage is expressed in retarding, attenuating, or even warding off the insidious changes of the target cells which are implicated in atherogenesis. As has been just shown, IL-33's salutary activities rely on modifications of the genetic responses inherent to atherogeneissusceptible cells rather than on direct prevention of ROSmediated cellular damage. Really, while in ex vivo experiments the impact of IL-33 on modifying transcription of the genetic information has been proved beyond any doubt, direct interaction of IL-33 with ROS-mediated reactions in the process of atherogenesis seems as much as excluded.

\section{IL-33 AND GENE EXPRESSION IN ADIPOSE TISSUE}

Lipid-modulating effects of IL-33 have been shown to extend from individual cells, notably subendothelial macrophages, to a compact tissue, namely the white adipose tissue (WAT). Even in normal conditions, adipose tissue has the capacity to perform distinct immune reactions, being considered as an antecedent of the phylogenetically younger, more sophisticated immune organs such as the thymus, the spleen 
and the lymph nodes. Persons affected by obesity display a low-grade inflammation which is highly reminiscent of, if not identical with that which underlies atherogenesis. In obesity, just the same as in atherosclerosis-prone vessels, WAT is infiltrated by inflammatory/immune cells beginning, once again, by T-lymphocytes and macrophages. Developmentally, adipocytes and macrophages stem from a common precursor. Accordingly, these inflammatory cells, along with resident adipocytes, produce a vast array of proinflammatory cytokines and chemokines. Of note, WATinfiltrating T-lymphocytes exhibit the Th1 pattern of activation with predominant IFN- $\gamma$ production. The prevalent Th1 immune responses in WAT are operative at the expense of $\mathrm{T}_{\text {reg }}$ or Th2 responses, both of them known anti-inflammatory reactions whose activities are thus substantially diminished. From among WAT-infiltrating macrophages, both $\mathrm{F} 4 / 80^{+}$and $\mathrm{F} 4 / 80^{-}$cells express the ST2L receptor, thereby manifesting a potential to respond to IL-33 signaling. Moreover, IL-33 is expressed in human adipose tissue [35]. As it is, IL-33 may perform in WAT the role of a metabolic regulator, as has been shown in a recent study by Miller and colleagues [36].

The authors showed that treatment of adipose tissue cultures with IL-33 induced the production of Th2 cytokines IL-5, IL-10 and IL-13, and chemokines MCP-1, MIP-1a, and KC. At the same time, expression of adipogenic and metabolic genes was decreased. A Th1-to-Th2 shift of the immune response can be inferred from this finding, paralleling the inhibition of atherogenesis including the absence of any IL-33-mediated effect in ST2 ${ }^{-/-}$cells [37].

Addition of IL-33 to differentiating murine SVF cells (i.e., stromal vascular fraction, a part of WAT which is made up predominantly by interstitial and vascular cells with some scattered adipocytes) significantly inhibited lipid accumulation. The mechanisms of decreased lipid content in SVF are attributable to decreased mRNA expression of:

i. $\mathbf{C} / \mathbf{E B P} \boldsymbol{\alpha}$ (CAAT enhancer-binding protein- $\alpha$ ),

ii. SREBP-1c (sterol regulatory element-binding protein 1c),

iii. liver X receptors (LXR)- $\boldsymbol{\alpha}, \mathbf{L X R}-\boldsymbol{\beta}$, and PPAR- $\boldsymbol{\gamma}$,

all of them transcription factors that are required for proper adipogenesis [38].

\section{IL-33 AND ALTERNATIVE ACTIVATION OF MAC- ROPHAGES}

These in vitro findings were confirmed and extended in in vivo experiments performed in spontaneously obese $(o b / o b)$ mice. The animals were administered IL-33 by the intraperitoneal route. As compared to controls, the surplus of IL-33 did not affect the body weight in $o b / o b$ mice; however, most adipocytes decreased in size. Furthermore, IL-33 exposure significantly attenuated total serum cholesterol levels, but it did not impact either on high-density lipoprotein cholesterol or on triglyceride concentrations. Of note, increased total IL-33 amount also increased the number of alternatively activated M2 macrophages, a subtype which is able to perform voluble anti-inflammatory effects [39]. This activity of IL-33 was executed in liver macrophages/Kupffer cells of the $a b / o b$ mice by increasing the expression and supporting the stability of mRNA implicated in the production of distinct M2 markers, such as L-arginase ( $\operatorname{Arg} 1)$, Ym1 (Chi313), RELM $\alpha /$ Fizz1 (retnla or resistin-like molecule alpha/found in inflammatory zone 1).

Expression of the peroxisome proliferator-activated receptor- $\delta$ (PPAR- $\delta$ ) protein, which too is involved in macrophage M2 polarization, was simultaneously increased in the liver of IL-33-treated $o b / o b$ mice [40]. On the other hand, no significant change was observed in the expression of M1 markers, such as inducible nitric oxide synthase (i-NOS) [41].

\section{PRO-INFLAMMATORY ACTIVITIES OF IL-33}

Notwithstanding the above-referred findings, it must be now explicitly stated that IL-33 is in no wise an unidirectional anti-inflammatory mediator._Rather, its activities branch out into different - sometimes seemingly contradictory - results, in accordance with actual cellular and cytokine-dependent microenvironmental conditions.

Bourgeois and coworkers [42] showed in their study that the capacity to promote a pro-Th1 cytokine profile constitutes a general feature of IL-33 applying likewise to C57BI/6 and BALB/c mice, which are biased towards a pro-Th1 and a pro-Th2 response, respectively. However, the exact mechanism by which IL-33 increases cytokine production by iNKT or NK cells remains obscure. Apparently, an interaction with the ST2 receptor alone is not sufficient to initiate IFN- $\gamma$ secretion by IL-33. IFN- $\gamma$ production does not occur in the absence of TCR or IL-12 signaling if cell cultures are set up with naive iNKT $\left(\mathrm{CD}^{+} \mathrm{NK} 1.1^{+}\right)$or NK cells. The same holds true for IL-4 production, implying that IL-33 acts as a cofactor rather than an inducer per se. Somewhat paradoxically to the hypothesis just mentioned, IL-33 administration alone proved to activate both iNKT and NK cells in vivo, which must have occurred indirectly, by way of endogenous factors generated by other target cells of IL-33. This is consistent with the fact that IFN- $\gamma$ could only be detected in response to IL-33 alone, i.e. not aided by IL-12, in cultures set up with whole spleen cells. These data are in agreement with the notion that IL-33 can contribute as a costimulatory factor to innate cellular immune responses.

The ability of IL-33 to contribute to IFN- $\gamma$ production, thereby supporting Th1-profiled inflammatory/immune responses, was complemented by additional studies. Yang and colleagues [43] reported that Th1 cytotoxic cells (Tc1), when cultured in vitro, produced unexpectedly high amounts of the ST2 receptor. This expression of ST2 relied on T-bet, the master Th1/Tc1 transcription factor which governs the Th1profiled inflammatory/immune response. Consequently, IL33 along with IL-12, supports IFN- $\gamma$ production by Tc1 cells. These results expand a hitherto unknown role of IL-33, i.e. that of promoting effector type 1 adaptive immune responses on top of innate immune responses.

All these findings seem to be in accord with another recently published study [44] which itself is in direct contradiction to the two afore-mentioned studies $[18,36]$. The authors of this particular study showed in HUVEC isolated from fresh umbilical cords and in HCAEC from coronary arteries from patients undergoing heart transplantation that 
were cultivated with or without rhIL-33 that IL-33 evoked increased adhesion of human PBMC and monocytes via increased E-selectin, VCAM-1 and ICAM-1. These effects of IL-33 were comparable to those of TNF- $\alpha$. At the same time, IL- 6 and IL- 8 were increased by IL-33. This effect occurred at the level of both mRNA and respective proteins production. Surprisingly, in this study, IL-33 was unable to increase the production of IL-4, IL-5 or IL-10. As is the case with all members of the IL-1 family, IL-33 increased NF- $\kappa$ B nuclear translocation. Moreover, in human carotid endarterectomy specimens, the authors found IL-33 and ST2 colocalized with von Willebrand factor in atherosclerotic tissue. All the effects of IL-33 were concentration-dependent, being highest with a significant increase at concentration from 1 to $100 \mathrm{ng} / \mathrm{mL}$ of IL-33.

\section{IL-33 AS AN ALARMIN}

IL-33 is implicated in the pathogenesis of many diseases, most of which exhibit the involvement of Th2 lymphocytes and/or the Th2 cytokine profile [45]. The precise source of IL-33 remains so far unclear. By contrast to remaining members of the IL-1 superfamily, primordial source of IL-33 has not been traced to immune cells which have been stimulated via pattern recognition receptors. Instead, IL-33 appears to be constitutively produced by endothelial cells and fibroblasts where high amounts of the mediator are present within the nuclei $[46,47]$. Also in contrast to other IL-1 family counterparts, IL-33 is produced as a full-length molecule that is already biologically active. No previous cleaving by caspase 1 is needed. In many aspects, IL-33 is close to IL-1 $\alpha$. Both of the two are biologically active as full-length molecules and, of note, both are present in the nuclei of the producing cells. [48]. IL-33 binding to the nucleus reflects two different biological properties. IL-33, just the same as IL-1 $\alpha$, performs the role of an alarmin. In unfavorable conditions, it is expelled out of damaged cells in order to alert the immune system on imminent danger. Both IL-33 and IL- $1 \alpha$ thus resemble the prototypic alarmin high mobility group box 1 (HMGB-1). As an alarmin, IL-33 is operative in a paracrine fashion so as to initiate sterile inflammation. Within the nucleus, IL-33 associates with heterochromatin, henceforth negatively affecting gene transcription. It is of paramount importance to state here that this activity occurs in an intracrine, non-IL-33 receptor-mediated fashion. This fact is the more striking when compared with all the studies in which IL-33 exercised its effects solely in the presence of the ST2 receptor. An illustrative example of this IL-33 duality (paracrine versus intrakrine activities) can be summarized in human monocytes [49]. In cells that have been stimulated via E. coli LPS (a TLR4 agonist) and P.gingivalis LPS (a TLR2 agonist), IL-33 mRNA and protein are found in the cytoplasm, with no evidence of nuclear translocation. No IL33 secretion occurs. By contrast, in monocytes undergoing apoptosis, IL-33 is sequestered in the nucleus. However, it is released into the extracellular space once the LPS-stimulated monocytes succumb to necrosis.

\section{IL-33 AND THE TRANSCRIPTION FACTOR NF-KB}

IL-33 interacts with the transcription factor NF- $\kappa$ B and dampens its activity. In endothelial cells, endogenous IL-33 was shown to interact with heterochromatin and mitotic chromosomes, i.e. with cells undergoing division and growth, but to a comparable degree in the nuclei of nonproliferating endothelial cells. Nuclear localization of ectopically expressed IL-33 depends on a homeodomain-like helix-turn-helix motif in the N-terminus (aa 1-65) of IL-33. The N-terminal region in full length IL-33 (aa 1-65 in human IL-33) is responsible for nuclear translocation and interaction with the heterochromatin. This region also functions as a chromatin binding motif (CBM, aa 40-58) [50]. This motif mediates IL-33 binding to histone H2A-H2B dimers on the nucleosomes, thus altering chromatin architecture. This mechanism is thought to be responsible of the IL-33 ability to repress gene transcription homeodomain-like HTH motif, which was reported to be necessary for nuclear accumulation and transcriptional repression [47].

Inflammatory processes including those initiated by IL33 are amplified by the transcription factor NF-кB. IL-33 in its nuclear position binds to the NF- $\mathrm{B}$ p65 subunit both in the cytosol and in the nucleus of the producing cell. The resulting IL-33+NF- $\kappa$ B p65 protein-protein interaction impedes the NF- $\mathrm{BB}$-dependent transcription by two mechanisms: [i.] decreased binding of nuclear DNA by NF- $\kappa \mathrm{B}$ and [ii.] reduced p65-mediated transactivation. This effect may be due to the ability of IL-33 to deny recruitment of further molecules required for transactivation. The constitutive binding of p65 by IL-33 is only weak in unstimulated cells. However, this binding is mightily increased upon stimulation with IL-1 $\beta$. Collectively, IL-33 interacts with the p65 region that is critically needed for the interaction with $\mathbf{I} \mathbf{k} \mathbf{B} \boldsymbol{\alpha}$, an

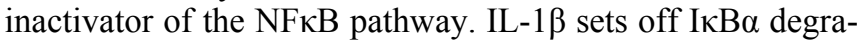
dation which results in enhanced IL-33/p65 interactions. The interaction of p65 with IL-33 also had biological consequences in cellular responses to rhIL-1 $\beta$ stimulation. Prototypic NF- $\kappa \mathrm{B}$ response genes like $I \kappa B \alpha, T N F-\alpha$, and $C-R E L$ were negatively affected in their expression by the presence of IL-33, whereas the expression of housekeeping genes remained unaffected. Not only that the reported repressive activity of full length IL-33 on gene expression is a general phenomenon, as suggested previously, but that, in addition, IL-33 interferes with the regulation of NF- $\kappa B$ target genes. Stimulation with saturating concentrations of IL-1 $\beta$ is able to overcome the dampening effects of IL-33, implying that IL33 attenuates NF- $\mathrm{B}$ activity that is evoked by suboptimal stimuli. IL-33 performs the best NF- $\kappa B$-downregulating activity in settings where relatively few p65 molecules are set free from the complex with I $\kappa \mathrm{B} \alpha$. Accordingly, overexpression of p65 in amounts that exceed the inhibitory capacity of $I \kappa \mathrm{B} \alpha$, also avoids inhibition by IL-33. This suggests that strong impulses originating from the activation of many p65 subunits can overwhelm the attenuating IL-33 effect and permit the induction of unrestrained NF- $\kappa B$ activity [46].

\section{IL-33 IN ENDOTHELIAL CELLS}

Endothelial cells in their position between individual organs and circulating blood are exposed to unremitting signals from the bloodstream. This blood-evoked perturbations, albeit most of them only of fable intensity, nonetheless generate short pulses of NF- $\mathrm{BB}$ stimulation. In any case, this is an undesirable effect which may result in unwanted activation of inflammatory processes. Under physiological condi- 
tions, nuclear IL-33 has the ability to dampen such tonic NF$\kappa \mathrm{B}$ signals. Strong activation of NF- $\kappa \mathrm{B}$ signaling in endothelial cells, however, will override the inhibitory effect of IL33 and result in upregulation of adhesion molecules, cytokines, and other inflammatory mediators. On the other hand, pro-inflammatory stimuli cause downregulation of nuclear IL-33 in quiescent endothelial cells [51]. Alternatively, severe cell damage can lead to the release of IL-33, which can then act as an alarmin $[52,53]$. Thus, the same cytokine IL-33 in this case - can function in different intracellular and extracellular pathways to initiate, control, and terminate the inflammatory/innate immunity response [54].

\section{CONCLUSION}

On the basis of results from different studies which have been reviewed here, it may be soundly concluded that interleukin-33, a "dual-acting" or "Janus-face" cytokine from the IL-1 superfamily, appears to be protective against atherosclerosis. This effect, however, is not brought about in the form of a classically acting cytokine but, according to data presented in this article, rather in its form of an alarmin, which is a way leaving out the receptor ST2. The atheroprotective effect of the whole IL-33/ST2L axis may be exercised by increasing the production of natural IgM antibodies. This IL33 hypothesis needs to be corroborated by further studies.

\section{ACKNOWLEDGEMENT}

This work was supported by Charles University in Prague, Faculty of Medicine, Hradec Kralove, project "Prvouk".

\section{CONFLICT OF INTEREST}

The author(s) confirm that this article content has no conflicts of interest.

\section{REFERENCES}

[1] Grundtman, C.; Wick, G. The autoimmune concept of atherosclerosis. Curr. Opin. Lipidol., 2011, 22 (5), 327-334.

[2] Shibata, N.; Glass, C. K. Regulation of macrophage function in inflammation and atherosclerosis. J. Lipid Res., 2009, (50 Suppl), S277-S281.

[3] Yan, Z. Q.; Hansson, G. K. Innate immunity, macrophage activation, and atherosclerosis. Immunol. Rev., 2007, 219), 187-203.

[4] Hansson, G. K.; Hermansson, A. The immune system in atherosclerosis. Nat. Immunol., 2011, 12 (3), 204-212.

[5] Seneviratne, A.N.; Sivagurunathan, B.; Monaco, C. Toll-like receptors and macrophage activation in atherosclerosis. Clin. Chim. Acta, 2012, 413 (1-2), 3-14.

[6] Schulte, S.; Sukhova, G. K.; Libby, P. Genetically programmed biases in Th1 and Th2 immune responses modulate atherogenesis. Am. J. Pathol., 2008, 172 (6), 1500-1508.

[7] Baekkevold, E. S.; Roussigne, M.; Yamanaka, T.; Johansen, F. E.; Jahnsen, F. L.; Amalric, F.; Brandtzaeg, P.; Erard, M.; Haraldsen, G.; Girard, J. P. Molecular characterization of NF-HEV, a nuclear factor preferentially expressed in human high endothelial venules. Am. J. Pathol., 2003, 163 (1), 69-79.

[8] Schmitz, J.; Owyang, A.; Oldham, E.; Song, Y.; Murphy, E.; McClanahan, T. K.; Zurawski, G.; Moshrefi, M.; Qin, J.; Li, X.; Gorman, D.M.; Bazan, J.F.; Kastelein, R.A. IL-33, an interleukin1-like cytokine that signals via the IL-1 receptor-related protein ST2 and induces T helper type 2-associated cytokines. Immunity., 2005, 23 (5), 479-490.

[9] Talabot-Ayer, D.; Lamacchia, C.; Gabay, C.; Palmer, G. Interleukin-33 is biologically active independently of caspase- 1 cleavage. J. Biol. Chem., 2009, 284 (29), 19420-19426.
[10] Luthi, A.U.; Cullen, S.P.; McNeela, E.A.; Duriez, P.J.; Afonina, I.S.; Sheridan, C.; Brumatti, G.; Taylor, R.C.; Kersse, K.; Vandenabeele, P.; Lavelle, E.C.; Martin, S.J. Suppression of interleukin-33 bioactivity through proteolysis by apoptotic caspases. Immunity., 2009, 31 (1), 84-98.

[11] Enoksson, M.; Lyberg, K.; Moller-Westerberg, C.; Fallon, P. G.; Nilsson, G.; Lunderius-Andersson, C. Mast cells as sensors of cell injury through IL-33 recognition. J. Immunol., 2011, 186 (4), 25232528.

[12] Chackerian, A.A.; Oldham, E.R.; Murphy, E.E.; Schmitz, J.; Pflanz, S.; Kastelein, R. A. IL-1 receptor accessory protein and ST2 comprise the IL-33 receptor complex. J. Immunol., 2007, 179 (4), 2551-2555.

[13] Bulek, K.; Swaidani, S.; Qin, J.; Lu, Y.; Gulen, M.F.; Herjan, T.; Min, B.; Kastelein, R. A.; Aronica, M.; Kosz-Vnenchak, M.; Li, X. The essential role of single Ig IL-1 receptor-related molecule/Toll IL-1R8 in regulation of Th2 immune response. J. Immunol., 2009, 182 (5), 2601-2609.

[14] Kurowska-S. M.; Hueber, A.; Stolarski, B.; McInnes, I. B. Interleukin-33: a novel mediator with a role in distinct disease pathologies. J. Intern. Med., 2011, 269 (1), 29-35.

[15] Millonig, G.; Malcom, G. T.; Wick, G. Early inflammatory-immunological lesions in juvenile atherosclerosis from the Pathobiological Determinants of Atherosclerosis in Youth (PDAY)study. Atherosclerosis, 2002, 160 (2), 441-448.

[16] Ghosh, S.; Zhao, B.; Bie, J.; Song, J. Macrophage cholesteryl ester mobilization and atherosclerosis. Vascul. Pharmacol., 2010, 52 (12), 1-10.

[17] Turnquist, H. R.; Zhao, Z.; Rosborough, B. R.; Liu, Q.; Castellaneta, A.; Isse, K.; Wang, Z.; Lang, M.; Stolz, D. B.; Zheng, X. X.; Demetris, A. J.; Liew, F. Y.; Wood, K. J.; Thomson, A. W. IL-33 expands suppressive CD11b+ Gr-1(int) and regulatory $\mathrm{T}$ cells, including ST2L+ Foxp3+ cells, and mediates regulatory $\mathrm{T}$ cell-dependent promotion of cardiac allograft survival. J. Immunol., 2011, 187 (9), 4598-4610.

[18] McLaren, J.E.; Michael, D.R.; Salter, R.C.; Ashlin, T.G.; Calder, C.J.; Miller, A.M.; Liew, F.Y.; Ramji, D.P. IL-33 reduces macrophage foam cell formation. J. Immunol., 2010, 185 (2), 12221229.

[19] Seimon, T.; Tabas, I. Mechanisms and consequences of macrophage apoptosis in atherosclerosis. J. Lipid Res., 2009, 50, S382S387.

[20] Berland, R.; Wortis, H. H. Origins and functions of B-1 cells with notes on the role of CD5. Annu. Rev. Immunol., 2002, 20, 253-300.

[21] Lutz, H. U.; Binder, C. J.; Kaveri, S. Naturally occurring autoantibodies in homeostasis and disease. Trends Immunol., 2009, 30 (1), 43-51.

[22] Binder, C.J.; Shaw, P.X.; Chang, M.K.; Boullier, A.; Hartvigsen, K.; Horkko, S.; Miller, Y.I.; Woelkers, D.A.; Corr, M.; Witztum, J.L. The role of natural antibodies in atherogenesis. J. Lipid Res., 2005, 46 (7), 1353-1363.

[23] Lewis, M.J.; Malik, T.H.; Ehrenstein, M.R.; Boyle, J.J.; Botto, M.; Haskard, D.O. Immunoglobulin $\mathrm{M}$ is required for protection against atherosclerosis in low-density lipoprotein receptor-deficient mice. Circulation, 2009, 120 (5), 417-426.

[24] Chang, M.K.; Binder, C.J.; Miller, Y.I.; Subbanagounder, G.; Silverman, G.J.; Berliner, J.A.; Witztum, J.L. Apoptotic cells with oxidation-specific epitopes are immunogenic and proinflammatory. J. Exp. Med., 2004, 200 (11), 1359-1370.

[25] Shaw, P.X.; Horkko, S.; Chang, M.K.; Curtiss, L.K.; Palinski, W.; Silverman, G.J.; Witztum, J.L. Natural antibodies with the T15 idiotype may act in atherosclerosis, apoptotic clearance, and protective immunity. J. Clin. Invest, 2000, 105 (12), 1731-1740.

[26] Komai-Koma, M.; Gilchrist, D.S.; McKenzie, A.N.; Goodyear, C.S.; Xu, D.; Liew, F.Y. IL-33 activates B1 cells and exacerbates contact sensitivity. J. Immunol., 2011, 186 (4), 2584-2591.

[27] Sampi, M.; Ukkola, O.; Paivansalo, M.; Kesaniemi, Y.A.; Binder, C.J.; Horkko, S. Plasma interleukin-5 levels are related to antibodies binding to oxidized low-density lipoprotein and to decreased subclinical atherosclerosis. J. Am. Coll. Cardiol., 2008, 52 (17), 1370-1378.

[28] McLaren, J.E.; Ramji, D.P. Interferon gamma: a master regulator of atherosclerosis. Cytokine Growth Factor Rev., 2009, 20 (2), 125135. 
[29] Fitzgerald, M.L.; Mujawar, Z.; Tamehiro, N. ABC transporters, atherosclerosis and inflammation. Atherosclerosis, 2010, 211 (2), 361-370.

[30] Betters, J.L.; Yu, L. NPC1L1 and cholesterol transport. FEBS Lett., 2010, 584 (13), 2740-2747.

[31] Stefanovic-Racic, M.; Perdomo, G.; Mantell, B.S.; Sipula, I.J.; Brown, N.F.; O'Doherty, R.M.A moderate increase in carnitine palmitoyltransferase 1a activity is sufficient to substantially reduce hepatic triglyceride levels. Am. J. Physiol Endocrinol. Metab, 2008, 294 (5), E969-E977.

[32] Listenberger, L.L.; Ostermeyer-Fay, A.G.; Goldberg, E.B.; Brown, W.J.; Brown, D.A. Adipocyte differentiation-related protein reduces the lipid droplet association of adipose triglyceride lipase and slows triacylglycerol turnover. J. Lipid Res., 2007, 48 (12), 2751-2761.

[33] Aragane, K.; Fujinami, K.; Kojima, K.; Kusunoki, J. ACAT inhibitor F-1394 prevents intimal hyperplasia induced by balloon injury in rabbits. J. Lipid Res., 2001, 42 (4), 480-488.

[34] Connelly, M.A.; Williams, D.L. SR-BI and cholesterol uptake into steroidogenic cells. Trends Endocrinol. Metab, 2003, 14 (10), 467472.

[35] Wood, I.S.; Wang, B.; Trayhurn, P. IL-33, a recently identified interleukin-1 gene family member, is expressed in human adipocytes. Biochem. Biophys. Res. Commun., 2009, 384 (1), 105-109.

[36] Miller, A.M.; Asquith, D.L.; Hueber, A.J.; Anderson, L.A.; Holmes, W.M.; McKenzie, A.N.; Xu, D.; Sattar, N.; McInnes, I.B.; Liew, F.Y. Interleukin-33 induces protective effects in adipose tissue inflammation during obesity in mice. Circ. Res., 2010, 107 (5), 650-658.

[37] Nishimura, S.; Manabe, I.; Nagasaki, M.; Eto, K.; Yamashita, H.; Ohsugi, M.; Otsu, M.; Hara, K.; Ueki, K.; Sugiura, S.; Yoshimura, K.; Kadowaki, T.; Nagai, R. CD8+ effector T cells contribute to macrophage recruitment and adipose tissue inflammation in obesity. Nat. Med., 2009, 15 (8), 914-920.

[38] Gustafson, B.; Hammarstedt, A.; Andersson, C.X.; Smith, U. Inflamed adipose tissue: a culprit underlying the metabolic syndrome and atherosclerosis. Arterioscler. Thromb. Vasc. Biol., 2007, 27 (11), 2276-2283.

[39] Gordon, S.; Martinez, F.O. Alternative activation of macrophages: mechanism and functions. Immunity., 2010, 32 (5), 593-604.

[40] Chawla, A. Control of macrophage activation and function by PPARs. Circ. Res., 2010, 106 (10), 1559-1569.

[41] Martinez, F.O.; Gordon, S.; Locati, M.; Mantovani, A. Transcriptional profiling of the human monocyte-to-macrophage differentiation and polarization: new molecules and patterns of gene expression. J. Immunol., 2006, 177 (10), 7303-7311.

[42] Bourgeois, E.; Van, L.P.; Samson, M.; Diem, S.; Barra, A.; Roga, S.; Gombert, J. M.; Schneider, E.; Dy, M.; Gourdy, P.; Girard, J.P.; Herbelin, A. The pro-Th2 cytokine IL-33 directly interacts with invariant NKT and NK cells to induce IFN-gamma production. Eur. J. Immunol., 2009, 39 (4), 1046-1055.

[43] Yang, Q.; Li, G.; Zhu, Y.; Liu, L.; Chen, E.; Turnquist, H.; Zhang, X.; Finn, O. J.; Chen, X.; Lu, B. IL-33 synergizes with TCR and
IL-12 signaling to promote the effector function of CD8+ T cells. Eur. J. Immunol., 2011, 41 (11), 3351-3360.

[44] Demyanets, S.; Konya, V.; Kastl, S.P.; Kaun, C.; Rauscher, S.; Niessner, A.; Pentz, R.; Pfaffenberger, S.; Rychli, K.; Lemberger, C.E.; de, M.R.; Heinemann, A.; Huk, I.; Groger, M.; Maurer, G.; Huber, K.; Wojta, J. Interleukin-33 induces expression of adhesion molecules and inflammatory activation in human endothelial cells and in human atherosclerotic plaques. Arterioscler. Thromb. Vasc. Biol., 2011, 31 (9), 2080-2089.

[45] Kurowska-Stolarska, M.; Stolarski, B.; Kewin, P.; Murphy, G.; Corrigan, C. J.; Ying, S.; Pitman, N.; Mirchandani, A.; Rana, B.; van, R. N.; Shepherd, M.; McSharry, C.; McInnes, I. B.; Xu, D.; Liew, F. Y. IL-33 amplifies the polarization of alternatively activated macrophages that contribute to airway inflammation. J. Immunol., 2009, 183 (10), 6469-6477.

[46] Ali, S.; Mohs, A.; Thomas, M.; Klare, J.; Ross, R.; Schmitz, M. L.; Martin, M. U. The dual function cytokine IL-33 interacts with the transcription factor NF-kappaB to dampen NF-kappaB-stimulated gene transcription. J. Immunol., 2011, 187 (4), 1609-1616.

[47] Roussel, L.; Erard, M.; Cayrol, C.; Girard, J. P. Molecular mimicry between IL-33 and KSHV for attachment to chromatin through the H2A-H2B acidic pocket. EMBO Rep., 2008, 9 (10), 1006-1012.

[48] Ali, S.; Nguyen, D.Q.; Falk, W.; Martin, M.U. Caspase 3 inactivates biologically active full length interleukin-33 as a classical cytokine but does not prohibit nuclear translocation. Biochem. Biophys. Res. Commun., 2010, 391 (3), 1512-1516.

[49] Nile, C.J.; Barksby, E.; Jitprasertwong, P.; Preshaw, P.M.; Taylor, J.J. Expression and regulation of interleukin-33 in human monocytes. Immunology, 2010, 130 (2), 172-180.

[50] Sue, S.C.; Dyson, H.J. Interaction of the IkappaBalpha C-terminal PEST sequence with NF-kappaB: insights into the inhibition of NF-kappaB DNA binding by IkappaBalpha. J. Mol. Biol., 2009, $388(4), 824-838$.

[51] Kuchler, A.M.; Pollheimer, J.; Balogh, J.; Sponheim, J.; Manley, L.; Sorensen, D.R.; De Angelis, P.M.; Scott, H.; Haraldsen, G. Nuclear interleukin-33 is generally expressed in resting endothelium but rapidly lost upon angiogenic or proinflammatory activation. Am. J. Pathol., 2008, 173 (4), 1229-1242.

[52] Carriere, V.; Roussel, L.; Ortega, N.; Lacorre, D.A.; Americh, L.; Aguilar, L.; Bouche, G.; Girard, J. P. IL-33, the IL-1-like cytokine ligand for ST2 receptor, is a chromatin-associated nuclear factor in vivo. Proc. Natl. Acad. Sci. U. S. A, 2007, 104 (1), 282-287.

[53] Moussion, C.; Ortega, N.; Girard, J.P. The IL-1-like cytokine IL-33 is constitutively expressed in the nucleus of endothelial cells and epithelial cells in vivo: a novel 'alarmin'? PLoS One, 2008, 3 (10), e3331.

[54] Oboki, K.; Ohno, T.; Kajiwara, N.; Arae, K.; Morita, H.; Ishii, A.; Nambu, A.; Abe, T.; Kiyonari, H.; Matsumoto, K.; Sudo, K.; Okumura, K.; Saito, H.; Nakae, S. IL-33 is a crucial amplifier of innate rather than acquired immunity. Proc. Natl. Acad. Sci. U. S $A, \mathbf{2 0 1 0}, 107$ (43), 18581-18586. 